\title{
Wavelet-based Reflection Symmetry Detection via Textural and Color Histograms: Algorithm and Results
}

\author{
Mohamed Elawady, Christophe Ducottet, Olivier Alata, Cécile Barat \\ Université de Lyon, UJM-Saint-Etienne, CNRS, IOGS, \\ Laboratoire Hubert Curien UMR5516, F-42023, Saint-Etienne, France \\ mohamed.elawady@univ-st-etienne.fr \\ Philippe Colantoni \\ Université de Lyon, UJM-Saint-Etienne, CIEREC EA n n 3068 , \\ Saint-Etienne, France
}

\section{Introduction}

The proposed algorithm detects globally the symmetry axes inside an image plane. The main steps are as follows: We firstly extract edge features using Log-Gabor filters with different scales and orientations. Afterwards, we use the edge characteristics associated with the textural and color information as symmetrical weights for voting triangulation. In the end, we construct a polar-based voting histogram based on the accumulation of the symmetry contribution (local texture and color information), in order to find the maximum peaks presenting as candidates of the primary symmetry axes.

Our contribution is twofold. Firstly, we extract edge features upon the application of Log-Gabor filters for symmetry detection instead of using Gabor filters. Secondly, we propose a similarity measure based on color image information, to improve the symmetry magnitude estimation in the voting step. In addition, we evaluate the proposed methods over all public datasets for reflection symmetry (single and multiple cases), in comparison with state-of-the-art algorithms.

The remaining part of the paper is organized as follows. In section 2, we explain Log-Gabor transform and its feature-based application on a gray-scale image. In section 3 , textural and color histograms of window-based features are described in details. In section 4 , we illustrate the detection of symmetry candidates through triangulation and voting processes. Experimental details and results are presented in section 5. Finally, section 6 contains conclusion and future work.

\section{Log-Gabor Edge Detection}

Log-Gabor filter consists of logarithmic transformation of a Gabor filter in the Fourier domain, which suppresses the negative effect of the DC component:

$$
\begin{gathered}
\hat{G}(\eta, \alpha ; s, o)=\hat{G}_{s}(\eta) \hat{G}_{o}(\alpha) \\
\hat{G}_{s}(\eta)=\exp \left(-\frac{\left(\log \left(\frac{\eta}{\eta_{s}}\right)\right)^{2}}{2\left(\log \left(\sigma_{\eta}\right)\right)^{2}}\right) U(\eta) \\
\hat{G}_{o}(\alpha)=\exp \left(-\frac{\left|\operatorname{atan}\left(\frac{\sin \left(\alpha-\alpha_{o}\right)}{\cos \left(\alpha-\alpha_{o}\right)}\right)\right|}{2 \sigma_{\alpha}^{2}}\right)
\end{gathered}
$$

where $(\eta, \alpha)$ are the log-polar coordinates representing radial and angular components over $S$ scales and $O$ orientations, associated with the frequency centers $\left(\eta_{s}, \alpha_{o}\right)$ and their bandwidths $\left(\sigma_{\eta}, \sigma_{\alpha}\right) . \hat{G}_{s}(\eta)$ is multiplied by low-pass Butterworth filter $U(\eta)$ of order 15 , and frequency 0.45 , to eliminate any extra frequency at Fourier corners.

The modulus of complex wavelet coefficients $I_{s, o}(x, y)$ are computed on an image $I$ (width $W$ and height $H$ ) over multiple scales $s \in\{1, \ldots, S\}$ and orientations $o \in$ $\left\{\frac{z \pi}{O}, z=0, \ldots, O-1\right\}$ as follows:

$$
\begin{gathered}
I \underset{G S}{\rightarrow} I_{G S} \stackrel{F T}{\rightarrow} \hat{I}_{G S} \\
I_{s, o}(x, y)=\left|F T^{-1}\left(\hat{I}_{G S} \times \hat{G}\right)\right|
\end{gathered}
$$

where $\hat{I}_{G S}$ is the gray-scale version of the image $I$ in frequency domain, and $F T(),. F T^{-1}($.$) are the Fourier trans-$ form and its inverse.

Figure 1 presents an example of Log-Gabor response $I_{s, o}$ on a natural image with a blurring background. Amplitude map $J(x, y)=\max _{s, o} I_{s, o}(x, y)$ highlights the edge details of the foreground object in a sharp way, accompanied by precise angular values in the corresponding orientation map $\phi(x, y)$. Upon a spatial sampling of the input image $I$ using non-interleaved cells along a regular grid (stride and 


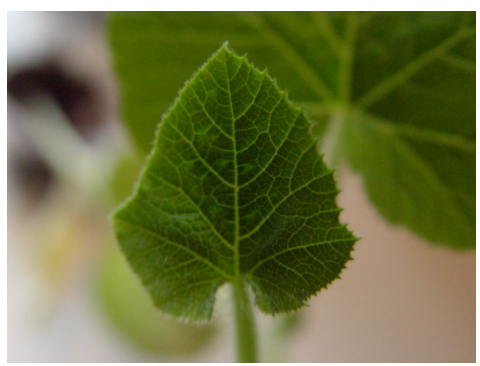

(a) Input

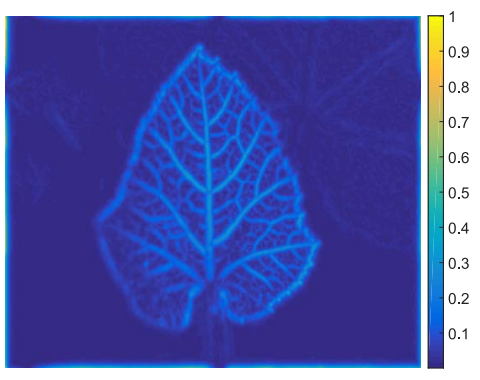

(b) Amplitude

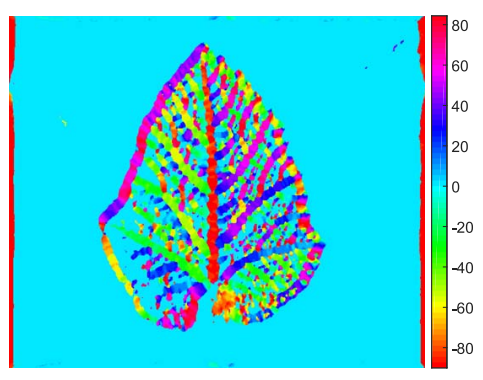

(c) Orientation

Figure 1: Log-Gabor response: (a) Input image. (b) Amplitude map $J \in[0,1]$. (c) Orientation map $\phi \in\left[-90^{\circ}, 90^{\circ}\right]$.

cell size are proportional to the maximum image dimension $\max (W, H)$ ). A feature point $p^{i}$ is extracted within each cell $D_{i}$ containing edges using the wavelet response of LogGabor filter $I_{s, o}\left(D_{i}\right)$, associated with its maximum wavelet response $J_{i}=J\left(p^{i}\right)$ along side with the corresponding orientation $\phi_{i}$ and color in $H S V$ color space $\psi_{i}$.

\section{Textural and Color Histograms}

The textural and color information around an edge segment are prominent similarity characteristics for natural images, describing the symmetrical behavior of local edge orientation, and the balanced distribution of luminance and chrominance components. Hence, we introduce two histograms: Firstly, neighboring textural histogram $h^{i}$ of size $N$ :

$$
\begin{gathered}
h^{i}(n)=\sum_{r \in D_{i}} J_{r} \mathbb{1}_{\Phi_{n}}\left(\phi_{r}\right), \\
\Phi_{n}=\left[\frac{n \pi}{N}, \frac{(n+1) \pi}{N}[, n=0, \ldots, N-1\right.
\end{gathered}
$$

where $\mathbb{1}$ is the indicator function. $h^{i}$ is $l 1$ normalized and circular shifted with respect to the orientation of the maximum magnitude $\phi_{i}$ among the neighborhood cell $D_{i}$. Secondly, the local $H S V$ histogram $g^{i}$ of size $C$ with subsampling rate $\left(C^{h u}: C^{s a}: C^{v a}\right)$

$$
\begin{aligned}
& g^{i}(c)=\sum_{r \in D_{i}^{*}} \mathbb{1}_{\Psi_{c}}\left(\psi_{r}\right), \\
& c=\left(c^{h u}, c^{s a}, c^{v a}\right), \\
& c^{h u} \in\left\{0, \ldots, C^{h u}-1\right\}, \\
& c^{s a} \in\left\{0, \ldots, C^{s a}-1\right\}, \\
& c^{v a} \in\left\{0, \ldots, C^{v a}-1\right\}, \\
& \Psi_{c}=\left[\frac{2 c^{h u} \pi}{C^{h u}}, \frac{2\left(c^{h u}+1\right) \pi}{C^{h u}}\left[\times\left[\frac{c^{s a}}{C^{s a}}, \frac{c^{s a}+1}{C^{s a}}\left[\times\left[\frac{c^{v a}}{C^{v a}}, \frac{c^{v a}+1}{C^{v a}}[\right.\right.\right.\right.\right.
\end{aligned}
$$

where $D_{i}^{*}$ is the neighborhood window around feature point $p^{i}, \psi_{c}$ is a sub-sampled set of $H S V$ space, in terms of three components: hue ( $h u)$, saturation $(s a)$ and value $(v a)$. $l 1$ normalization is applied to $g^{i}($.$) for bin-wise histogram$ comparison.

\section{Symmetry Triangulation and Voting}

A set of feature pairs $\left(p^{i}, p^{j}\right)$ of size $\frac{P(P-1)}{2}$ are elected such that $i \neq j$, and $P$ is the number of feature points. Then, we compute the symmetry candidate axis based a triangulation process with respect to the image origin. This candidate axis is parametrized by angle $\theta_{i, j}$ (orientation of the bisector of the pair segment $\left(p^{i}, p^{j}\right)$ ), and displacement $\rho_{i, j}$ (distance of the image origin to this bisector) and has a symmetry weight $\omega_{i, j}$ defined as follows:

$$
\begin{gathered}
\omega_{i, j}=\omega\left(p^{i}, p^{j}\right)=m(i, j) t(i, j) q(i, j) \\
m(i, j)=\left|\tau^{i} R\left(T_{i j}^{\perp}\right) \tau^{j}\right| \\
t(i, j)=\sum_{n=1}^{N} \min \left(h^{i}(n), \tilde{h}^{j}(n)\right) \\
q(i, j)=\sum_{c=1}^{C} \min \left(g^{i}(c), g^{j}(c)\right)
\end{gathered}
$$

where $\tau^{i}=\left[\cos \left(\phi_{i}\right), \sin \left(\phi_{i}\right)\right]^{T}, R\left(T_{i j}^{\perp}\right)$ is the reflection matrix with respect to the perpendicular of the line connecting $\left(p^{i}, p^{j}\right)$ [3, 4], and $\tilde{h}^{j}$ is the reverse version of $h^{j}$ histogram. $l 1$ normalization is applied to symmetry weights $\omega$.

A symmetry histogram $H(\rho, \theta)$ is defined as the sum of the symmetry weights of all pairs of feature points such as:

$$
H(\rho, \theta)=\sum_{\substack{p^{i}, p^{j} \\ i \neq j}} \omega_{i, j} \delta_{\rho-\rho_{i, j}} \delta_{\theta-\theta_{i, j}}
$$

where $\delta$ is the Kronecker delta.

the voting histogram $H(\rho, \theta)$ is smoothed using a Gaussian kernel to output a proper density representation, in 
which the major symmetry peaks are selected by reachingout clear extreme spots using well-known non-maximal suppression technique $[1,6]$. The spatial boundaries of each symmetry axis is computed through the convex hull of the associated voting pairs.

\section{Results and Discussions}

This section describes the details of public symmetry datasets, evaluation metrics, and experimental settings for performance comparison of the proposed work.

\subsection{Datasets description}

Five public datasets of reflection symmetry detection are used from three different databases: (1) PSU datasets (single, multiple): Liu's vision group proposed symmetry groundtruth for Flickr images (\# images = \# symmetries = 157 for single case, \# images = 142 and \# symmetries $=479$ for multiple case) in ECCV2010 ${ }^{1}$, CVPR2011 ${ }^{2}$ and CVPR2013 $^{3}$. (2) AVA dataset (single): Elawady et. al [4] provided axis groundtruth ${ }^{4}$ for some professional photographs $(\#$ images $=\#$ symmetries $=253$ for single case $)$ from large-scale aesthetic-based dataset called AVA [7]. (3) NY datasets (single, multiple): Cicconet et al. [2] introduced a symmetry database $(\#$ images $=\#$ symmetries $=176$ for single case, \# images = 63 and \# symmetries $=188$ for multiple case) in $2016^{5}$, providing more stable groundtruth.

\subsection{Evaluation metrics}

Assuming a symmetry line defined by two endpoints $\left(a=\left[a_{x}, b_{y}\right]^{T}, b=\left[b_{x}, b_{y}\right]^{T}\right)$, quantitative comparisons are fundamentally performed by considering a detected symmetry candidate $S C=\left[a^{S C}, b^{S C}\right]^{T}$ as a true positive (TP) respect to the corresponding groundtruth $G T=$ $\left[a^{G T}, b^{G T}\right]^{T}$ if satisfying the following two conditions:

$$
\begin{aligned}
& T\left(\operatorname{atan}\left(\frac{\operatorname{abs}\left(\left|\begin{array}{cc}
v_{x}^{S C} & v_{x}^{G T} \\
v_{y}^{S C} & v_{y}^{G T}
\end{array}\right|\right.}{<v^{S C}, v^{G T}>}\right)\right)<\gamma, \\
& \sqrt{\left(t_{x}^{S C}-t_{x}^{G T}\right)^{2}+\left(t_{y}^{S C}-t_{y}^{G T}\right)^{2}}<\zeta, \\
& v^{S C}=\left(a^{S C}-b^{S C}\right), v^{G T}=\left(a^{G T}-b^{G T}\right), \\
& t^{S C}=\frac{\left(a^{S C}+b^{S C}\right)}{2}, t^{G T}=\frac{\left(a^{G T}+b^{G T}\right)}{2}, \\
& T(\Gamma)= \begin{cases}\pi-\Gamma, & \text { if } \Gamma>\frac{\pi}{2} \\
\Gamma, & \text { otherwise }\end{cases}
\end{aligned}
$$

\footnotetext{
${ }^{1} \mathrm{http}: / /$ vision.cse.psu.edu/research/symmetryCompetition/index.shtml

${ }^{2} \mathrm{http} / / /$ vision.cse.psu.edu/research/symmComp/index.shtml

${ }^{3} \mathrm{http} / / / \mathrm{vision} . c s e . p s u . e d u /$ research/symComp13/content.html

${ }^{4}$ http://github.com/mawady/AvaSym

${ }^{5}$ http://symmetry.cs.nyu.edu/
}

The conditions represent angular and distance constraints between detected and groundtruth axes. These constraints are upper-bounded by the corresponding thresholds $\gamma$ and $\zeta$, which are defined in table 1 . Furthermore, the precision and recall rates are defined by selecting the symmetry peaks according to the candidates' amplitude normalized by the highest detection score, to show the performance curve for each algorithm. In addition, we used the maximum $F_{1}$ score identifying a unique comparison measure, to show the overall accuracy of each symmetry algorithm.

Table 1: Threshold values of evaluation metrics across different reflection symmetry competitions.

\begin{tabular}{c|c|c}
\hline \hline Competitions & $\gamma$ & $\zeta$ \\
\hline \hline CVPR2011 [8] & $10^{\circ}$ & $20 \% \times \operatorname{len}(G T)$ \\
CVPR2013 [5] & $10^{\circ}$ & $20 \% \times \min \{\operatorname{len}(M T), \operatorname{len}(G T)\}$ \\
\hline \hline
\end{tabular}

\subsection{Experimental settings}

We compare the proposed methods ( $L g$ : without color information and $\mathrm{LgC}$ : with color information) against three state-of-the-art approaches: Loy and Eklundh (Loy2006) [6], Cicconet et al. (Cic2014) [3], and Elawady et al. (Ela2016) [4]). Their source codes are used with default parameter values for performance comparison. In Log-Gabor edge detection, we set the number of scales $S$ and number of orientations $O$ to 12 and 32 . We also set the radial bandwidth $\sigma_{\eta}$ to 0.55 and the angular bandwidth $\sigma_{\alpha}$ to 0.2 . In textural and color histogram calculations, we define the number of bins for textural $N$ and color $C$ to 32 and 32 (sampling rate $C^{h u}: C^{s a}: C^{v a}=8: 2: 2$ ) respectively. In case of gray-scale images, contrast values are used instead of color information in $H S V$ color space. In symmetry voting, we declare the $2 \mathrm{D}$ histogram space of $\rho=\sqrt{W^{2}+H^{2}}$ displacement bins and $\theta=360$ orientation bins for extrema selection.

\subsection{Performance analysis}

In our experimental evaluation, the algorithms are executed to detect and compare the global symmetries inside synthetic and real-world images. Table 2 shows the true positive rates for the proposed methods ( $\mathrm{Lg}$ and $\mathrm{LgC}$ ) against Loy and Eklundh (Loy) [6], Cicconet et al. (Cic) [3], and Elawady et al. (Ela) [4]. $\mathrm{LgC}$ performs the best result among most cases in single and multiple symmetry, due to the importance of color information for the voting computations in colorful images. At the same time, $L g$ has the top 2 nd result, and sometimes the top 1st results in gray-scale or low-saturated images. Ela [4] ranked as the top 3rd result, due to the utilization of small grids to compute windowbased features. Thanks for the advantage of SIFT features, 


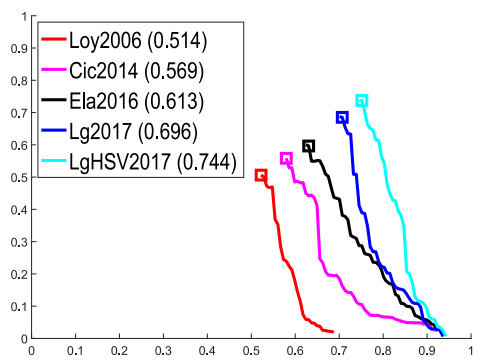

(a) PSU

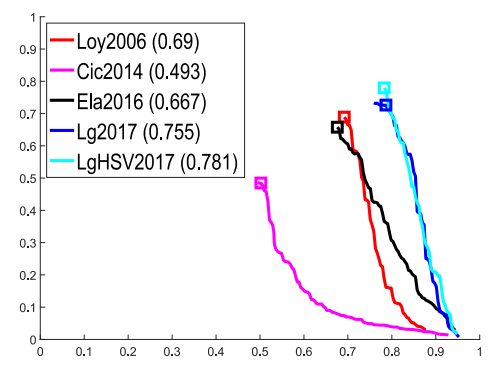

(b) AVA

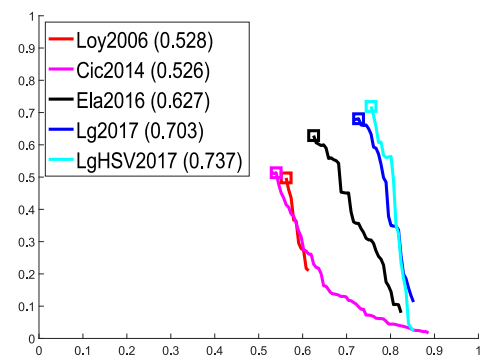

(c) NY

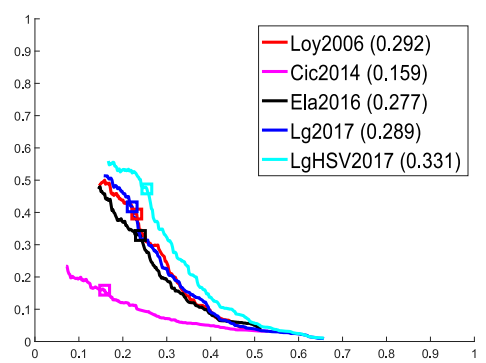

(d) PSUm

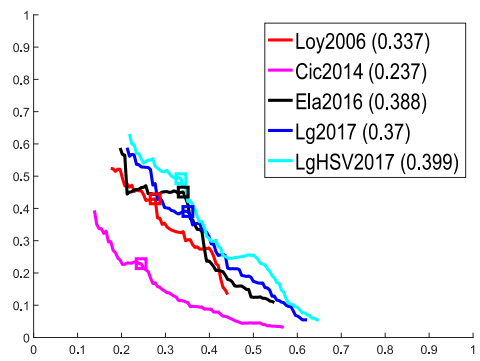

(e) NYm

Figure 2: Using evaluation metrics CVPR2013 [5], Precision-Recall curves on: (1) four single-case symmetry (a,b,c) datasets, and (2) three multiple-case symmetry $(\mathrm{d}, \mathrm{e})$ datasets to show the overall superior performance of our proposed methods ( Lg2017 and LgHSV2017) against the three prior algorithms (Loy2006 [6], Cic2014 [3], and Ela2016 [4]). The maximum F1 scores are qualitatively presented as square symbols along the curves, and quantitatively indicated between parentheses inside the top-right legends. Best seen on screen (zoom-in for details).

Table 2: Using evaluation metrics CVPR2013 [5], comparison of the true positive rates based on top detection for the proposed methods against the state-of-art algorithms. Symmetry datasets are presented as: single-case (first 3 rows) and multiple-case (last 2 rows), highlighting between parenthesis the number of images for each dataset. Top 1st and 2nd values are in Bold and underlined respectively.

\begin{tabular}{c|c|c|c|c|c}
\hline \hline Datasets & Loy[6] & Cic $[3]$ & Ela $[4]$ & $\operatorname{Lg}$ & $\operatorname{LgC}$ \\
\hline \hline PSU(157) & 81 & 90 & 97 & $\underline{109}$ & $\mathbf{1 1 6}$ \\
AVA(253) & 174 & 124 & 170 & $\underline{191}$ & $\mathbf{1 9 7}$ \\
NY(176) & 98 & 92 & 109 & $\underline{125}$ & $\mathbf{1 3 2}$ \\
\hline PSUm(142) & 69 & 68 & 67 & $\underline{74}$ & $\mathbf{7 9}$ \\
NYm(63) & 32 & 36 & 36 & $\underline{39}$ & $\mathbf{4 0}$ \\
\hline \hline
\end{tabular}

Loy [6] is still strong competent to be ranked as the top 4th result in general. Cic [3] has the lowest performance.

Figure 2 presents performance results in terms of precision and recall curves for single-case and multiplecase symmetry datasets, plus values of the maximum $F_{1}$ score to measure the performance of the proposed algorithms ( Lg2017 and LgHSV2017) against Loy and Eklundh (Loy2006) [6], Cicconet et al. (Cic2014) [3], and
Elawady et al. (Ela2016) [4]. In single-case symmetry, our method $L g 2017$ outperforms the other concurrent algorithms (Loy2006, Cic2014, and Ela2016) in the context of using only gray-scale version of involved images. Furthermore, color version of our method $L g H S V 2017$ exploits slightly improvement over gray-scale one $\operatorname{Lg} 2017$. On the other hand, Only $L g H S V 2017$ has better precision performance among others in PSUm and NYm datasets, due to many local symmetry groundtruth existing inside multiplecase mirror axes.

\section{Conclusion}

In this paper, we detect global symmetry axes inside an image using the edge characteristics of Log-Gabor wavelet response. For the purpose of improving our results, we additionally use textural and color histograms as local symmetrical measure around edge features. We show that the proposed methods provide a great improvement over single and multiple symmetry cases in different datasets. Future work will focus on improving the voting representations, respect to the number of symmetry candidate pairs, resulting a precise selection of symmetrical axis peaks and their corresponding voting features. 


\section{References}

[1] J. Canny. A computational approach to edge detection. IEEE Transactions on pattern analysis and machine intelligence, (6):679-698, 1986. 3

[2] M. Cicconet, V. Birodkar, M. Lund, M. Werman, and D. Geiger. A convolutional approach to reflection symmetry. Pattern Recognition Letters, 2017. 3

[3] M. Cicconet, D. Geiger, K. C. Gunsalus, and M. Werman. Mirror symmetry histograms for capturing geometric properties in images. In Computer Vision and Pattern Recognition (CVPR), 2014 IEEE Conference on, pages 2981-2986. IEEE, 2014. 2, 3, 4

[4] M. Elawady, C. Barat, C. Ducottet, and P. Colantoni. Global bilateral symmetry detection using multiscale mirror histograms. In International Conference on Advanced Concepts for Intelligent Vision Systems, pages 14-24. Springer, 2016. 2, 3,4

[5] J. Liu, G. Slota, G. Zheng, Z. Wu, M. Park, S. Lee, I. Rauschert, and Y. Liu. Symmetry detection from realworld images competition 2013: Summary and results. In Computer Vision and Pattern Recognition Workshops (CVPRW), 2013 IEEE Conference on, pages 200-205. IEEE, 2013. 3, 4

[6] G. Loy and J.-O. Eklundh. Detecting symmetry and symmetric constellations of features. In Computer Vision-ECCV 2006, pages 508-521. Springer, 2006. 3, 4

[7] N. Murray, L. Marchesotti, and F. Perronnin. Ava: A largescale database for aesthetic visual analysis. In Computer Vision and Pattern Recognition (CVPR), 2012 IEEE Conference on, pages 2408-2415. IEEE, 2012. 3

[8] I. Rauschert, K. Brocklehurst, S. Kashyap, J. Liu, and Y. Liu. First symmetry detection competition: Summary and results. Technical report, Technical Report CSE11-012, Department of Computer Science and Engineering, The Pennsylvania State University, 2011. 3 